Proc. Indian Acad. Sci. (Chem. Sci.), Vol. 107, No. 2, April 1995, pp. 143-148.

(C) Printed in India.

\title{
Dual ASE from 7-diethylamino-4-trifluoromethylcoumarin (C35)
}

\author{
D SASTI KUMAR ${ }^{1}$ and V MASILAMANI*2 \\ ${ }^{1}$ Department of Physics, Regional Engineering College, Tiruchirappalli 620015, India \\ ${ }^{2}$ Department of Physics, Anna University, Madras 600025, India
}

MS received 3 October 1994; revised 9 March 1995

\begin{abstract}
The laser dye coumarin 35 (C35) exhibits a dual ASE band similar to coumarin $1(\mathrm{C} 1)$ and does not require specific solute-solvent interaction for the formation of dual ASE.
\end{abstract}

Keywords. Coumarin 35; fluorescence; dual ASE; solute-solvent interactions.

\section{Introduction}

The laser dye 7-diethylamino-4-methylcoumarin (C1) exhibits dual amplified spontaneous emission (ASE) band in certain solvents, though a single ASE band is expected from its fluorescence spectrum as it contains only one fluorescence maximum (Masilamani et al 1986; Korol'kova et al 1987; Yip and Wen 1991). One ASE band appears corresponding to the fluorescence band maximum as normally expected and another ASE appears at longer wavelengths for which no fluorescence peak is observed. The dye $\mathrm{Cl}$, in general, shows the dependence of specific solute-solvent interaction for the formation of the longer wavelength ASE. However this ASE is attributed to different mechanisms: formation of TICT-solvent exciplex due to interaction between the dye molecule and the solvent molecule containing a functional group (Masilamani et al 1986), hydrogen bonding interaction between the dye and the solvent (Korol'kova et al 1987; Yip and Wen 1991), or solvation of the dye (Yip and Wen 1991). We report here the observation of the dual ASE phenomenon in a structurally similar laser dye $\mathrm{C} 35$ and its emission characteristics. These results are compared with those of $\mathrm{C} 1$ in view of the above proposed mechanisms.

\section{Experimental}

A nitrogen laser $(100 \mathrm{~kW}, 10 \mathrm{~ns})$ was used as an excitation source and the dye solution was pumped in a transverse configuration. A four-side polished quartz cell was used and kept canted to avoid feedback. The wavelength maximum of the ASE band was measured using a constant deviation spectrometer which was calibrated with $\mathbf{H g}$ spectrum as a reference. The relative intensity of the ASE bands was measured with a photomultiplier (RCA 931)-oscilloscope combination.

The dye, 7-diethylamine-4-trifluoromethylcoumarin (C35) was purchased from Sigma Chemical Co., USA. The TLC test did not show the presence of any impurity.

\footnotetext{
* For correspondence
} 
The dye was used as received. The solvents used were the spectroscopic grade. The absorption and fluorescence spectra were recorded using a Hitachi U2000 spectrophotometer and a Kontron SFM 25 spectrofluorimeter respectively.

\section{Results and discussion}

The dye C35 exhibits a dual ASE band (figure 1) in solvents like hexane, heptane, octane and cyclohexane (table 1). In hexane, the ASE bands appear around $432 \mathrm{~nm}$ (of weak intensity) and $470 \mathrm{~nm}$. But the dye exhibits only one fluorescence band maximum around $431 \mathrm{~nm}(8 \mathrm{mM}$ concentration) which corresponds to the shorter wavelength ASE band (i.e. $432 \mathrm{~nm}$ ) (figure 2).

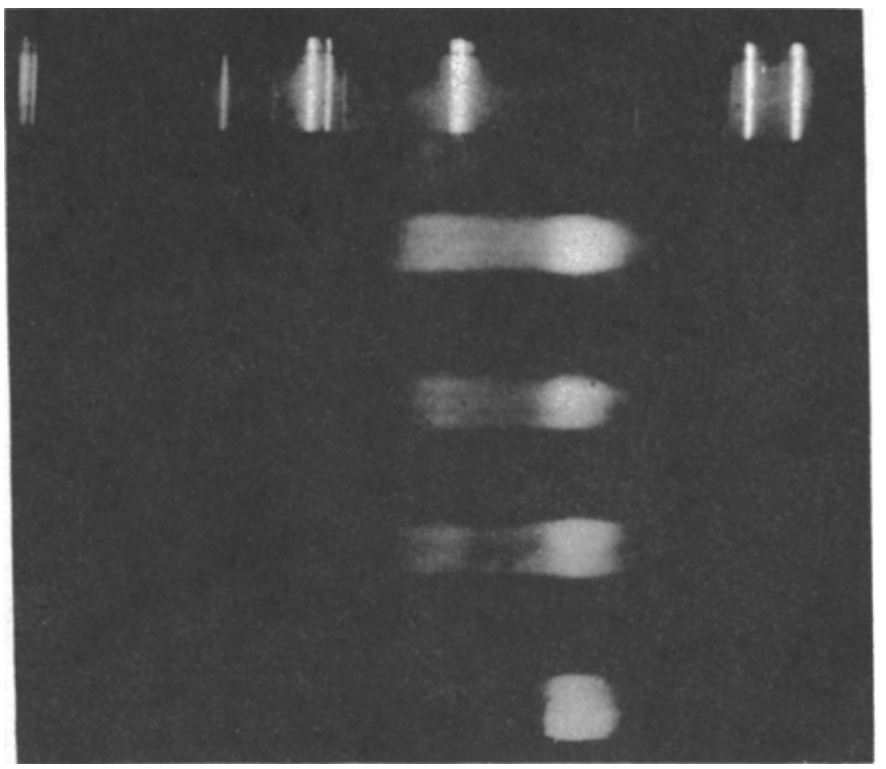

Figure 1. Spectrographic record of dual ASE band of C35 in (a) hexane, (b) heptane, (c) octane and (d) cyclohexane.

Table 1. Wavelength maxima of absorption, fluorescence and ASE bands of $\mathrm{C} 35$ in different solvents.

\begin{tabular}{lccc}
\hline Solvents & Absorption & Fluorescence & ASE \\
\hline Hexane & 375 & 428 & 432 and 470 \\
Heptane & 376 & 430 & 432 and 470 \\
Octane & 375 & 429 & 431 and 471 \\
Cyclohexane & 377 & 432 & 433 and 472
\end{tabular}

(Absorption and fluorescence for $8 \mu \mathrm{M}$ and ASE for $8 \mathrm{mM}$ concentrations) (wavelengths are in $\mathrm{nm}$ ) 


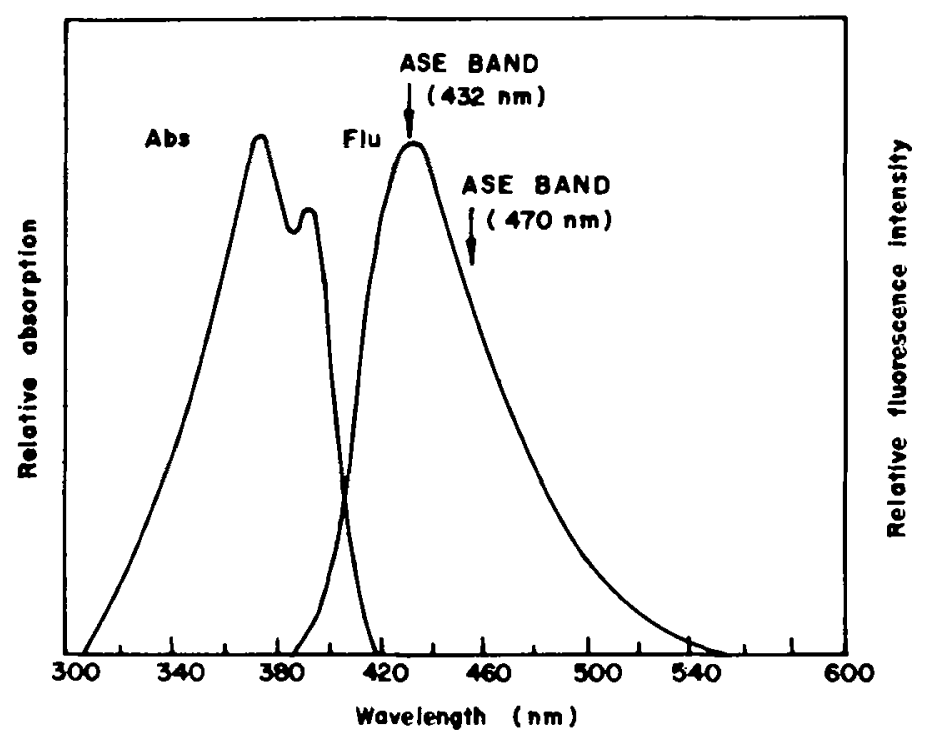

Figure 2. Absorption and fluorescence spectra of C35 in hexane for $8 \mathrm{mM}$ concentration.

The intensities of the longer wavelength $\operatorname{ASE}\left(I_{a}\right)$ and shorter wavelength $\operatorname{ASE}\left(I_{b}\right)$ vary with change in the concentration of the dye (figure 3 ). As the concentration is increased, the intensity of the longer wavelength ASE increases whereas the intensity of the shorter wavelength ASE decreases. However, the fluorescence spectrum always exhibits only one fluorescence maximum corresponding to the shorter wavelength ASE and undergoes a continuous red-shift as the concentration is increased. A similar red-shift is observed in the shorter wavelength ASE band with increase in the concentration whereas the longer wavelength ASE band remains almost the same.

The dye $\mathrm{Cl}$, which is similar to $\mathrm{C} 35$ in molecular structure with methyl group at the fourth position instead of trifluoromethyl group (figure 4), exhibits dual ASE in ethylacetate and isobutylmethylketone (table 2). The effect of concentration on the dual ASE of $\mathrm{Cl}$ is similar to $\mathrm{C} 35$ and the intensity change is more pronounced in C35 than in $\mathrm{Cl}$.

For $\mathrm{C} 35$ and $\mathrm{Cl}$, the $I_{a}$ and $I_{b}$ values also vary with a change in the temperature of the dye solution (figure 5). As the temperature is increased, the $I_{b}$ value increases whereas the $I_{a}$ value decreases. However, the fluorescence spectrum remains the same exhibiting only one band maximum.

The above results suggest that the longer wavelength ASE characteristics of C35 and $\mathrm{C} 1$ are similar and the species emitting the longer wavelength ASE are not detected using spectrofluorimeter measurements. Further the change in the ASE intensities with concentration and temperature suggests that the species emitting the longer wavelength ASE and shorter wavelength ASE are in dynamic equilibrium.

Many investigations have been carried out to understand the fluorescence characteristics of C1 and C35 (Jones et al 1980, 1985; Rettig and Klock 1985; Guo and Feng 1987; Yip and Wen 1990). Since $\mathrm{Cl}$ and $\mathrm{C} 35$ could undergo planar intramolecular charge transfer (ICT) and twisted intramolecular charge transfer (TICT) interactions in 


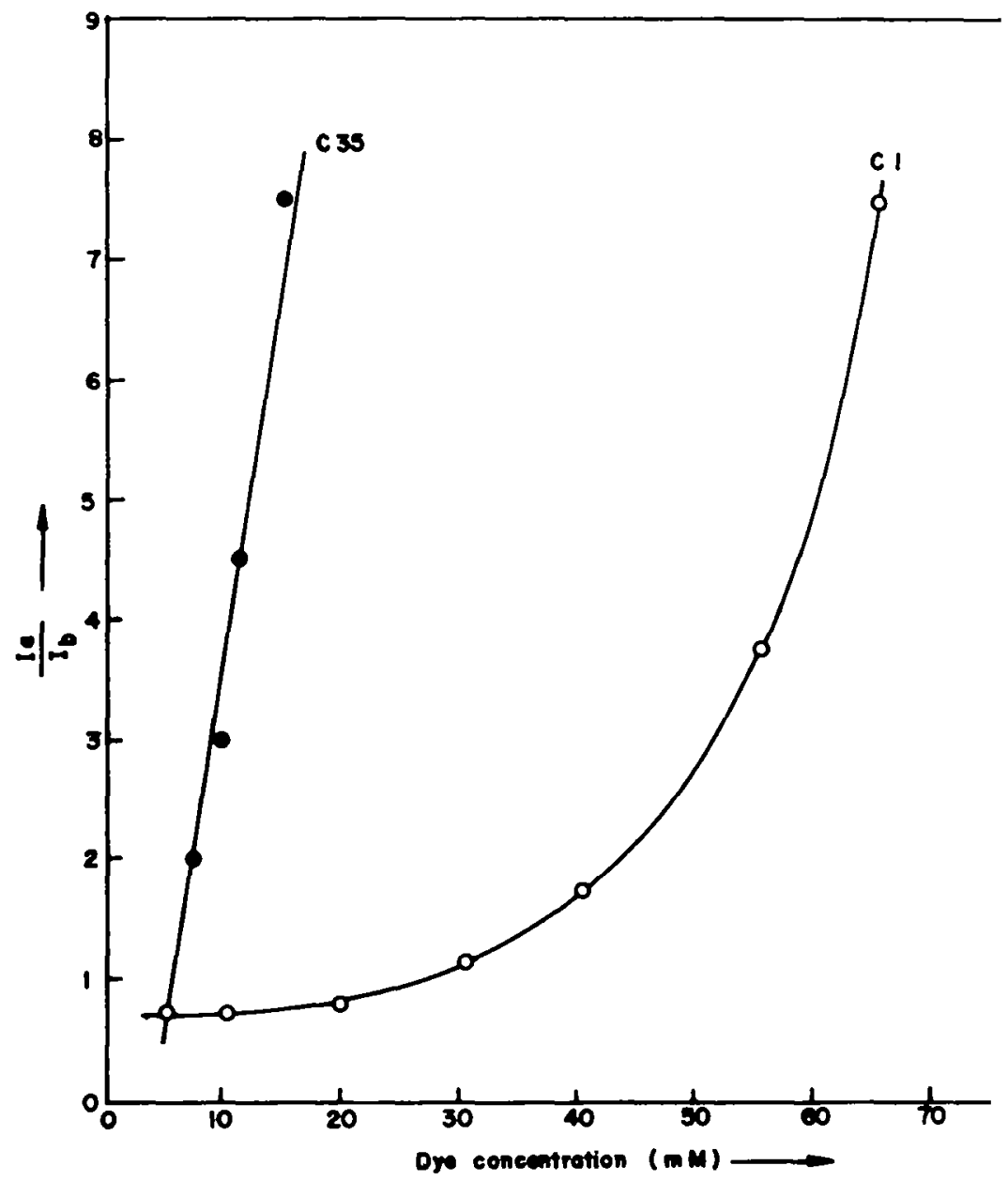

Figure 3. Effect of dye concentration on $I_{a} / I_{b}$. Cl in isobutylmethylketone anc in hexane.

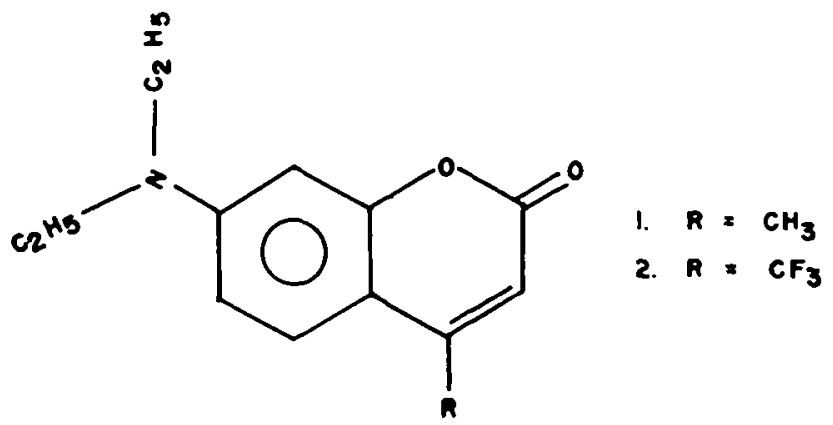

Figure 4. Molecular structures of (1) coumarin 1 and (2) coumarin 35. 
Table 2. Wavelength maxima of absorption, fluorescence and ASE bands of $\mathrm{Cl}$ in different solvents.

\begin{tabular}{lccc}
\hline Solvents & Absorption & Fluorescence & ASE \\
\hline Benzene & 359 & 410 & 415 \\
Toluene & 360 & 410 & 415 \\
Ethylacetate & 362 & 413 & 417 and 450 \\
isobutylmethylketone & 365 & 422 & 425 and 450
\end{tabular}

(Absorption and fluorescence for $8 \mu \mathrm{M}$ and ASE for $8 \mathrm{mM}$ concentrations) (Wavelengths are in $\mathbf{n m}$ )

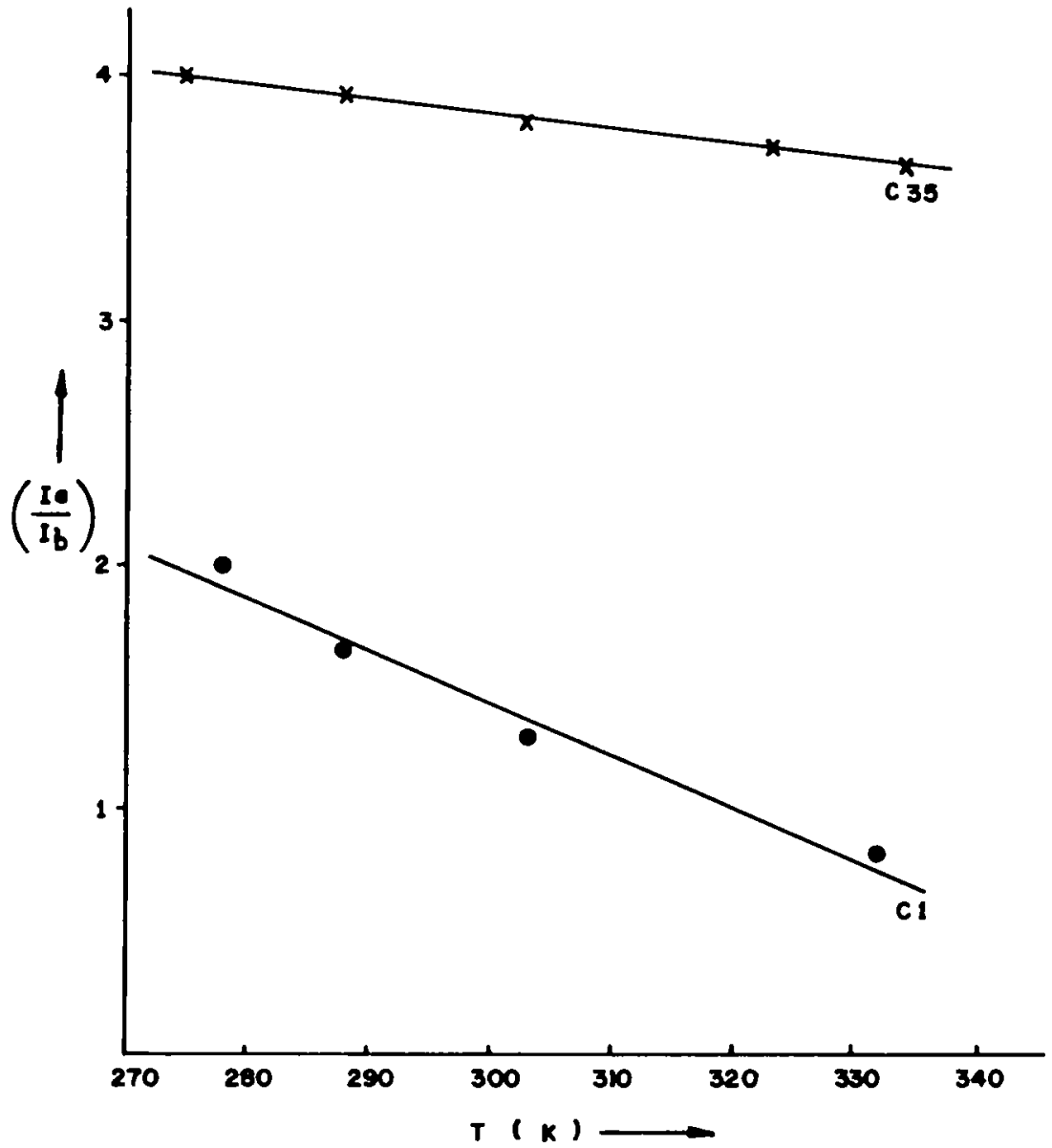

Figure 5. Plot of $I_{a} / I_{b}$ as a function of temperature, $\mathrm{C} 1$ in isobutylmethylketone and $\mathrm{C} 35$ in cyclohexane. 
the excited state (as proposed for dimethylaminobenzonitrile to explain its dual fluorescence (Grabowski et al 1979), the ICT-TICT model has been proposed to explain the dual ASE behaviour of $\mathrm{Cl}$ and $\mathrm{C} 35$, though these dyes exhibit only one fluorescence band maximum.

The dye $\mathrm{C} 1$ has been found to exhibit dual ASE in certain solvents (e.g. butyl acetate) containing functional groups or when a protic solvent (e.g. ethanol, water or butanol) or aprotic polar solvent (acetonitrile) is added to aprotic non-polar solvent (butyl acetate, dioxane or benzene). The dual ASE phenomenon observed in solvents containing functional groups is attributed to ICT planar and TICT-solvent exciplex (Masilamani et al 1986), and to $\mathrm{H}$-bonding in the case of protic and aprotic non-polar solvent mixtures (Korol'kova et al 1987; Yip and Wen 1991). In aprotic polar and aprotic non-polar solvent mixture, the dual ASE phenomenon is attributed to solvation (Yip and Wen 1991).

The dye C35 exhibits dual ASE in inert solvent environments. This indicates that the species emitting the longer wavelength ASE (in the case of C35) do not require specific solute-solvent interaction for their formation.

The dye $\mathrm{C} 35$ is more polar than $\mathrm{Cl}$ due to the presence of trifluoromethyl group substitution (Loboda et al 1984; Guo and Feng 1987). The higher polarity of the dye molecule appears to favour the longer wavelength ASE because longer wavelength ASE is observed for $\mathrm{C} 35$ even in an inert solvent like hexane. In contrast, $\mathrm{Cl}$ which is less polar than C35, requires the assistance of a solvent having a functional group for the stabilisation of the species emitting the longer wavelength ASE.

\section{Conclusion}

The appearance of dual ASE in inert solvents for C35 indicates that this dye does not require specific solute-solvent interaction for the formation of the longer wavelength ASE. The polarity of the dye molecule appears to influence the dual ASE characteristics.

\section{References}

Grabowski Z R, Rotkiewicz K, Siemiarczuk A, Cowley J and Baumann W 1979 Nouv. J. Chim. 3443

Guo C and Feog Y 1987 J. Chem. Soc., Faraday Trans. I 832533

Jones G II, Jackson W R, Chol-Yoo C and Bergmark W R 1985 J. Phys. Chem. 89294

Jones G II. Jackson W R and Halpern A M 1980a Chem. Phys. Lett. 72391

Jones G II, Jackson W R, Kanoktanaporn S and Halpern A M 1980b Opt. Commun. 33315

Korolkova N V, Reva M G and Uzhinov B M 1987 Sov. J. Quant. Electron. 17526

Loboda L I, Sokalova J V, Ya Il'chenko A and Koval'chuk R E 1984 J. Appl. Spectrosc. 40674

Masilamani V, Chandrasekar V, Sivaram B M, Sivasankar B and Natarajan S 1986 Opt. Commun. 59203

Rettig W and Klock A 1985 Can. J. Chem. 631649

Yip R W and Wen Y X $1990 \mathrm{~J}$. Photöchem. Photobiol. A54 263

Yip R W and Wen Y X 1991 Can. J. Chem. 691413 